\title{
Les trajectoires de passage à l'âge adulte en Europe
}

\author{
\begin{tabular}{l|l} 
& \\
Vitor Sérgio Ferreira & Institut des sciences sociales - Université de Lisbonne.
\end{tabular} \\ Cátia Nunes
}

Mots-clés : Déstandardisation - Transition vers l'âge adulte Comparaison européenne.

La déstandardisation des transitions du cours de la vie semble être un phénomène convergent et relativement répandu dans l'espace européen. Les marqueurs traditionnels du passage à l'âge adulte - tels que l'autonomie financière et résidentielle, le mariage ou avoir des enfants - sont de plus en plus longs et étendus dans le temps. Cependant, les comparaisons entre pays suggèrent l'existence de différences dans ces expériences de transition. Cet article vise à identifier la variété des voies de transition vers l'âge adulte en Europe. En quoi sont-elles le reflet des différents régimes de transition de la jeunesse? De plus, l'article tente de discuter dans quelle mesure la déstandardisation reflète également la déritualisation des marqueurs traditionnels du passage à l'âge adulte, en évaluant leur valeur ritualiste. À partir de l'European Social Survey, sont présentés et discutés les résultats sur les expériences et les valeurs symboliques d'événements traditionnellement perçus comme étant importants pour devenir adulte : le premier travail à temps complet, le départ du domicile parental, la première expérience conjugale et le premier enfant. Des différences entre les pays sont mises en évidence, offrant ainsi une présentation des diverses voies et attitudes sur le devenir adulte dans les sociétés européennes.

D ans un contexte fortement caractérisé par le risque, l'instabilité et l'incertitude, en l'absence de sécurité de l'emploi, de carrière prévisible, de relations durables et qui engagent, on peut à juste titre se demander si les mécanismes qui définissaient traditionnellement le fait de devenir adulte n'ont pas perdu leurs valeurs sociale et transitionnelle (Gauthier, 2000). Les marqueurs sociaux séparent-ils toujours de façon rituelle ceux qui les ont accomplis et les autres, et institutionnalisent-ils un statut différent, dans le sens où ils définiraient ceux qui sont adultes et les autres? (Bourdieu, 1982). Quelle est leur place dans le cadre cognitif et évaluatif des individus? Dans quelle mesure les marqueurs sociaux sont-ils au centre du processus de passage au statut d'adulte? Et peut-on s'attendre à ce que la déritualisation diffère d'un pays européen à l'autre? Des études comparatives font apparaître de grandes différences d'un pays à l'autre en ce qui concerne les trajectoires des jeunes et les valeurs normatives du passage à l'âge adulte. En effet, la pratique des marqueurs ritualisés traditionnels et la valeur qui leur est attachée ne reculent pas de manière homogène dans toutes les sociétés européennes. La manière dont ces marqueurs sont vécus et perçus subjectivement peut refléter différents contextes institutionnels et culturels. Ainsi, cet article s'appuie sur l'hypothèse selon laquelle, au lieu d'un processus général et uniforme de déstandardisation et de déritualisation, il est possible que I'on observe des variations et des différences en fonction des contextes et conditions historiques et institutionnels. Dans l'ensemble, les données empiriques indiquent que, loin de constituer une tendance générale qui englobe, uniformément, le passage à l'âge adulte, la déstandardisation et la déritualisation adoptent des formes et des rythmes distincts d'un pays à l'autre et d'une catégorie sociale à une autre. Elles acquièrent des formes et des contenus sociaux différents en fonction des conditions institutionnelles qui caractérisent le « régime de transition des jeunes » de chaque pays ou ensemble de pays (Walther, 2006) (encadré 1, p. 22).

Les recherches sociologiques transnationales mettent en évidence en Europe un grand nombre de différences dans l'expérience du passage à l'âge adulte pour les jeunes, tant en raison de l'existence de différents régimes institutionnalisés au niveau national que de la diversité intranationale des conditions de vie des jeunes. Les schémas de régulation institutionnelle varient considérablement d'un pays à l'autre (éducation,

Les auteurs remercient Andreas Walther, Barbara Stauber et deux experts anonymes pour leurs commentaires pertinents sur une version préliminaire du présent article. 
Encadré 1

"Le régime de transition de la jeunesse »

Le concept de "régime de transition de la jeunesse » (« regime of youth transitions »), développé par Andreas Walther et son équipe de I'EGRIS (European Group for Integrated Social Research), désigne non seulement une structure de conflits et d'intérêts historiquement appliquée par les acteurs sociaux sur la vie des jeunes et les périodes de cette vie (institutions, agences ou organisations définissant des règles qui tendent à façonner et à réguler les pratiques des jeunes dans un scénario institutionnel national donné), mais aussi un ensemble de valeurs et de représentations qu'ils reproduisent et conçoivent comme «normales» (ou comme constituant la norme) au sein de chaque contexte social ou national (comme, par exemple, I'âge donnant accès à certains domaines sociaux : l'âge auquel on entre à l'école, auquel on termine la scolarité obligatoire, auquel on commence à travailler, auquel on peut obtenir son permis de conduire, voter, fumer ou consommer de l'alcool, etc.). Ce concept s'inspire largement des travaux de Gösta Esping-Andersen, et en particulier de sa typologie des systèmes de protection sociale. Le sociologue commence, en 1990, par distinguer trois types de régimes (social-démocrate, conservateur et libéral) puis, dans ses travaux ultérieurs (1999), il identifie un régime supplémentaire : le régime de protection sociale méditerranéen.

formation, emploi, logement, système de protection sociale, participation à la vie civique, politique publique à l'égard des jeunes, etc.), mais aussi en fonction de la situation économique individuelle (plus ou moins développée et stable) et des conditions historicoculturelles (sexe, famille, appartenance ethnique, mobilité sociale, etc.). Ces aspects structurels viennent moduler les différents régimes de transition des jeunes, donnant lieu à des chronologies différentes (moments, séquences, nombre et combinaisons d'événements (Billari et al., 2000) entre les marqueurs de passage traditionnels à l'âge adulte, ainsi que différentes attitudes et valeurs qu'on leur attache, en fonction du contexte national auquel appartient telle ou telle génération de jeunes européens. Parallèlement, ces mêmes marqueurs de passage sont vécus et valorisés de manière très diverse par différents profils sociaux, et des variables telles que le sexe, la tranche d'âge, le niveau d'études et la pratique religieuse qui peuvent, elles aussi, influencer fortement ces différences d'attitudes.

Cet article présente succinctement des données empiriques sur les trajectoires différentes des tendances à la déstandardisation et à la déritualisation dans une perspective transnationale européenne. Il analyse dans quelle mesure les marqueurs traditionnels du passage à l'âge adulte ont perdu de leur valeur opérationnelle et symbolique en tant que marqueurs de transition. Ainsi, sont comparés différents régimes de transition des jeunes en Europe. Si I'on s'appuie sur les recherches antérieures, partielles et essentiellement qualitatives, on peut avancer que la déstandardisation et la déritualisation des processus de transition de la jeunesse sont probablement plus avancées dans les pays aux régimes de transition socio-démocrates ou universalistes que dans les pays aux régimes libéraux ou plus conservateurs. On peut également s'attendre à ce que, dans les pays qui se caractérisent par des régimes de transition sous-protecteurs, les processus de déstandardisation et de déritualisation ne soient pas aussi manifestes. Cette situation s'explique par le caractère rudimentaire de leur système de protection sociale et la forte dépendance vis-à-vis de la famille, d'une part, ainsi que par le pouvoir normatif de l'Église catholique, dont l'enseignement moral sur les valeurs familiales est plutôt strict, d'autre part. On peut dresser le même constat pour les anciens pays communistes. Si l'on pouvait penser que l'idéologie communiste, qui préconisait une intervention de l'État à grande échelle, était plutôt favorable à des trajectoires déstandardisées et déritualisées, dans la pratique, toutefois, la plupart des pays communistes se caractérisaient par de puissantes contraintes normatives pesant sur les individus, signe d'un niveau de déstandardisation et de déritualisation relativement faible. Et, après la chute du communisme, l'Église catholique est revenue en force dans certains de ces pays. Tenant compte de ces scénarios, cet article entend répondre, par le biais de la comparaison, à deux questions : dans quelle mesure la standardisation des principaux marqueurs sociaux relatifs à la transition à l'âge adulte recule-t-elle, objectivement, dans les pays européens? Et, dans quelle mesure la situation la plus déstandardisée s'accompagne-t-elle des processus les moins ritualisés de passage à l'âge adulte ? Cette analyse se penche d'abord sur le moment et l'ordre chronologique des événements qui définissent conventionnellement le passage à l'âge adulte. Ensuite, elle s'attache à la valeur symbolique attribuée à ces mêmes événements en tant que marqueurs sociaux de passage. Enfin, l'article observe les différences entre les pays et en leur sein, et présente les diverses attitudes vis-à-vis du passage à l'âge adulte.

\section{Littérature sur le passage à l'âge adulte}

Dans les sociétés industrielles occidentales, la vie était socialement organisée autour de systèmes $\mathrm{d}^{\prime}$ âge relativement stables, où se succédaient conventionnellement "l'enfance », " la jeunesse », "l'âge adulte » et "la vieillesse ». Le passage de I'un à l'autre était identifié à travers un ensemble 
de marqueurs de passage, c'est-à-dire des événements sociaux qui ont lieu dans les domaines institutionnels fondamentaux des sociétés occidentales (par exemple, école, marché du travail ou famille) et qui organisent de façon normative les périodes de la vie (Deschavanne et Tavoillot, 2007 ; Fournier, 2008 ; Guillemard, 2005). L'achèvement des études, l'entrée sur le marché du travail, le départ du foyer parental et la fondation d'une nouvelle famille, en vivant en couple et en devenant à son tour parent, constituent les marqueurs de passage les plus importants et normatifs de la transition à la vie d'adulte dans les sociétés occidentales, notamment en Europe. Traditionnellement, ces marqueurs étaient vécus dans un cadre temporel linéaire, selon un enchaînement d'événements organisés et prévisibles, qui passait par l'accomplissement successif de chaque marqueur social. Ces événements se produisaient selon un ordre chronologique et de façon définitive, à des moments qui différaient essentiellement en fonction de la classe sociale [Pais, 1993 ; Gomes (da) Silva, 1999] et du sexe [Fonseca (da), 2001 ; Moen, 1996 ; Stauber, 2006].

Plus récemment, la recherche sociologique a commencé à caractériser le passage à l'âge adulte comme un processus plus complexe, flexible, intermittent et pluriel. II ressort de plusieurs études que les moments et les séquences de ces marqueurs de passage sont de moins en moins stabilisés et organisés, et donc davantage déstandardisés. Le processus de déstandardisation correspond à la désynchronisation et au recul de l'uniformisation des moments et des séquences des événements de la vie, lesquels sont devenus plus dispersés et hétérogènes en termes d'âge et de durée (Brückner et Mayer, 2005). Le schéma de l'emploi durable, de I'avancement de la carrière, du mariage à un jeune âge et d'une vie familiale stable semble céder la place à des périodes et des vies plus mouvementées. En fait, la réalité sociale qui se dessine ouvre une nouvelle expérience sociale pour les jeunes, plus diffuse et labyrinthique, signe d'une société plus instable et plus incertaine. Cette déstandardisation des transitions pendant la jeunesse semble constituer un phénomène convergent et relativement répandu en Europe, surtout depuis l'effondrement des régimes communistes (Kovacheva, 2001 ; Nugin, 2008 ; Tomanovic et Ignjatovic, 2006 ; Vultur, 2005) et l'affaiblissement des systèmes d'emploi et de protection sociale censément universels qui caractérisent en particulier les pays du nord de l'Europe (Kugelberg, 1998). Dans le même temps, des recherches transnationales accumulent des données faisant apparaître, dans toute l'Europe, une tendance répandue à l'espacement des intervalles et à la remise à plus tard du moment où l'on atteint plusieurs marqueurs de passage (Billari et Wilson, 2001 ; Chambaz, 2001 ; Cicchelli, 2001 ;
Cicchelli et Bréviglieri, 2007 ; Cicchelli et al., 2006 ; Chisholm et al., 1995 et 1998 ; Chisholm et Pais, 1997 ; EGRIS, 2001 ; Moreno, 2008 ; Walther et al., 1999 ; Van de Velde, 2005). Cette tendance s'explique en raison, d'une part, de l'allongement des périodes d'études et de formation et, d'autre part, d'une entrée sur le marché du travail sans cesse repoussée et compromise pour ces jeunes qui connaissent de plus en plus de phases de chômage, et/ou de sous-emploi, d'emploi temporaire, ainsi que $d^{\prime} u n e$ forte rotation entre différentes formes d'emplois « atypiques » et précaires (Bradley et Devadason, 2008; Cachón Rodríguez, 2008 ; Ferreira et al., 1999 ; Ferreira, 2006 ; Müller et Gangl, 2003).

Outre ces nouvelles conditions sociales, certains suggèrent également que l'évolution générale qui touche la démographie et les valeurs conduit à la déstandardisation et à la complexité croissante des transitions vers I'âge adulte (Shanahan, 2000). La cohabitation, le fait de ne pas avoir d'enfant, le célibat ou la parentalité extraconjugale, par exemple, sont mieux acceptés et davantage pratiqués. D'autres événements, comme le mariage ou l'arrivée d'un enfant, non seulement sont retardés mais, bien souvent, se caractérisent par une inversion de leur séquentialité traditionnelle, voire par une disparition de leur interdépendance. On peut se marier puis ne pas avoir d'enfants ou avoir un enfant avant de se marier. Dans un contexte de profonde déstandardisation de la linéarité classique du passage à l'âge adulte, on pourrait avancer l'hypothèse de l'érosion concomitante de la valeur symbolique et normative des marqueurs et des rituels les plus institutionnels de ce passage. Ces rituels servaient à créer de la stabilité dans le cours de la vie sociale, mais ils renfermaient et exprimaient aussi une signification transitionnelle du passage au statut d'adulte. Cependant, lorsque la stabilité sociale qui en découle est ébranlée, la signification traditionnelle peut être réarrangée, voire disparaître, et la valeur symbolique au sein de l'espace social reculer. Ce processus, que I'on peut appeler "déritualisation »(Pais, 2001), correspond à une organisation moins normative du cours de la vie et entraîne l'effondrement de pratiques symboliques ritualisées, autrefois engagées pour les individus et les groupes. Si ce processus de déritualisation est surtout mis en évidence par la disparition des activités ritualisées traditionnelles qui se déroulent au cours de la vie, il peut aussi être révélé par la perte de la valeur transitionnelle traditionnelle de ces activités. Par conséquent, la déritualisation ne signifie pas immédiatement que les marqueurs sociaux traditionnels du passage sont abandonnés, mais plutôt que leur valeur transitionnelle en tant que marqueurs sociaux peut s'amenuiser et être perçue et valorisée différemment. Par ailleurs, cette déritualisation signifie également 
Encadré 2

\section{European Social Survey}

L'European Social Survey (ESS - www.europeansocialsurvey.org) est un réseau de chercheurs qui ont pour objectif d'observer les attitudes, les valeurs et les comportements dans une perspective longitudinale et transnationale. D'un point de vue méthodologique, ce projet suit une approche quantitative reposant sur une enquête menée tous les deux ans. La première édition de cette enquête remonte à 2002 et, depuis, trois autres ont été menées. Le questionnaire se compose de groupes de questions récurrentes d'une édition sur l'autre (l'exposition aux médias, la participation à la vie politique, l'immigration, la confiance interpersonnelle et la confiance dans les institutions, le bienêtre et les valeurs sociales) ainsi que de deux ou trois groupes de modules tournants. Pour chaque édition, des équipes multinationales de chercheurs sont formées afin de participer à l'élaboration des modules tournants du questionnaire. La troisième édition de I'ESS comportait un module tournant consacré aux événements de la vie et aux transitions intitulé «The timing of life: The organization of life course in Europe ». Il a été élaboré par Francesco Billari (Université Bocconi, Italie), Gunhill Hagestag (Adger University College, Norvège), Aart Liefbroer (Netherlands Interdisciplinary Demographic Institute et Université libre d'Amsterdam) et Zsolt Spéder (Hungarian Central Statistical Office)

que les jeunes sont aujourd'hui capables de définir et de choisir leurs propres marqueurs symboliques de passage : de nouveaux rites qui, dans le cadre de leur vie privée, montrent symboliquement qu'ils ont surmonté certains événements marquants de leur vie. Ce sont des "rites d'impasse » (« ritos de impasse ») (Ferreira, 2008 ; Pais, 2009), à savoir des initiatives volontaires et privées, associées aux événements imprévisibles auxquels sont de plus en plus soumises l'identité et les transitions des jeunes.

\section{Méthodologie : I'enquête de l'European Social Survey}

Si les résultats accumulés jusqu'ici sont quelque peu partiels et restreints, tant par le nombre de pays étudiés, les méthodes utilisées (pour la plupart qualitatives et reposant sur des informations secondaires), voire les domaines de la vie traités, le module tournant intitulé "The timing of life: the organisation of life course in Europe " ( $3^{\mathrm{e}}$ édition, 2006), de l'European Social Survey (ESS) (encadré 2) constitue une excellente opportunité de collecter des informations comparables, systématiques et à grande échelle sur les façons de vivre le passage à I'âge adulte dans différents pays européens. Par conséquent, pour la première fois, une enquête complète, reposant sur une méthode quantitative et fortement standardisée, a été menée sur ce sujet, rendant possible une analyse comparative transnationale et transculturelle des expériences, significations et attitudes relatives au passage à I'âge adulte en Europe. Au total, vingt-trois pays européens ont été systématiquement analysés et comparés (tableau 1) (1). Les données se rapportent à des échantillons représentatifs de la population de chaque pays participant, et les répondants sont âgés de 15 ans au minimum (pas de limite supérieure) (2). Elles ont été recueillies au moyen d'entretiens en face-à-face, qui ont eu lieu au domicile des répondants. Ces entretiens ont été soumis à une procédure de contrôle de la qualité et, en cas d'incohérences, les répondants ont été recontactés afin qu'ils valident des informations. Des variables de pondération ont également été calculées afin de corriger les différences entre les

Informations relatives aux échantillons, par pays

\begin{tabular}{|c|c|c|}
\hline & Nombre & $\begin{array}{c}\text { Erreur } \\
\text { d'échantillonnage } \\
(a=0,95)\end{array}$ \\
\hline Allemagne & 2916 & $\pm 1,8$ \\
\hline Autriche & 2405 & $\pm 2,0$ \\
\hline Belgique & 1798 & $\pm 2,3$ \\
\hline Bulgarie & 1400 & $\pm 2,6$ \\
\hline Chypre & 995 & $\pm 3,1$ \\
\hline Danemark & 1505 & $\pm 2,5$ \\
\hline Slovaquie & 1766 & $\pm 2,3$ \\
\hline Slovénie & 1476 & $\pm 2,6$ \\
\hline Espagne & 1875 & $\pm 2,3$ \\
\hline Estonie & 1517 & $\pm 2,5$ \\
\hline Finlande & 1896 & $\pm 2,3$ \\
\hline France & 1986 & $\pm 2,2$ \\
\hline Pays-Bas & 1889 & $\pm 2,3$ \\
\hline Hongrie & 1518 & $\pm 2,5$ \\
\hline Irlande & 1802 & $\pm 2,3$ \\
\hline Norvège & 1750 & $\pm 2,3$ \\
\hline Pologne & 1721 & $\pm 2,4$ \\
\hline Portugal & 2222 & $\pm 2,1$ \\
\hline Royaume-Uni & 2394 & $\pm 2,0$ \\
\hline Russie & 2437 & $\pm 2,0$ \\
\hline Suisse & 1804 & $\pm 2,3$ \\
\hline Suède & 1926 & $\pm 2,2$ \\
\hline Ukraine & 2002 & $\pm 2,2$ \\
\hline Total & 43000 & - \\
\hline
\end{tabular}

1) En tout, vingt-cing pays ont participé à la troisième édition de l'European Social Survey. Cependant, en raison de la date de publication des données et des informations relatives aux pondérations, la Lettonie et la Roumanie n’ont pas été incluses dans I'analyse.

(2) Pour de plus amples informations sur la conception de l'échantillonnage et les indicateurs d'évaluation de la qualité pour chaque pays, voir Matsuo et al., 2009. 
probabilités de sélection des répondants (pondération a priori) et d'ajuster la taille de l'échantillon à celle de la population réelle du pays en question (pondération démographique). Pour les analyses comparatives, ces deux variables ont été combinées (pondération a priori et pondération démographique) et activées.

L'article s'appuie sur l'analyse de deux ensembles d'indicateurs relatifs à la transition à l'âge adulte (tableau 2). Le premier ensemble s'intéresse spécifiquement à l'expérience des répondants vis-à-vis de certains marqueurs sociaux clés à cette étape de la vie : premier emploi à temps plein ; premier départ du foyer parental; première expérience de vie en couple (époux/partenaire) ; premier mariage et premier enfant. Les questions ont permis de déterminer l'âge auquel les répondants ont vécu chacun de ces événements pour la première fois. Le deuxième ensemble d'indicateurs s'éloigne des expériences individuelles pour s'attacher aux attitudes sociales, plus particulièrement à la valeur symbolique qui est attribuée à différents marqueurs sociaux concernant la transition à l'âge adulte, à savoir: I'autonomie résidentielle, l'emploi, la vie en couple et la parentalité.

Par conséquent, cette enquête a permis non seulement de rendre compte de l'expérience individuelle des marqueurs sociaux clés du passage à l'âge adulte, mais également d'élargir le champ de
I'analyse sur la manière dont les individus évaluent subjectivement la valeur symbolique de ces marqueurs. Elle ouvre une opportunité innovante et révolutionnaire de mesurer et de comparer des expériences et des attitudes relatives au passage à l'âge adulte en Europe, en englobant à la fois les dimensions objective et subjective susceptibles de jouer un rôle dans la forme des trajectoires de vie des individus.

\section{Moments auxquels les jeunes font l'expérience des marqueurs sociaux du passage à l'âge adulte}

Dans l'abondante littérature consacrée à ce sujet, la transition de l'école au marché du travail constitue l'un des marqueurs sociaux les plus étudiés pour le passage au statut d'adulte. Cet intérêt s'explique non seulement par le fait que ce facteur représente un passage fortement institutionnalisé, déterminé par des normes d'âge qui définissent juridiquement des droits et des devoirs (Neugarten et al., 1965), mais aussi par le fait que, dans une large mesure, les premiers pas des jeunes vers I'autonomie financière en dépendent. Les Européens connaissent leur première expérience professionnelle en moyenne à l'âge de 19 ans. Néanmoins, l'âge auquel on entre sur le marché du travail varie d'un pays européen à l'autre (graphique 1, p. 26) (3). Au Portugal, cette première

Tableau 2

Indicateurs de transition à l'âge adulte

\begin{tabular}{|c|c|c|}
\hline \multirow[t]{2}{*}{ Questions } & En quelle année... & $\begin{array}{l}\text { Pour être considéré comme un adulte, } \\
\text { quelle importance cela a-t-il... }\end{array}$ \\
\hline & $\begin{array}{l}\text { 1) avez-vous commencé à travailler (emploi rémunéré ou } \\
\text { apprentissage rémunéré d'au moins } 20 \text { heures par semaine } \\
\text { pendant au moins trois mois)? } \\
\text { 2) avez-vous quitté vos parents pendant au moins } \\
\text { deux mois pour commencer à vivre sans eux? } \\
\text { 3) avez-vous pour la première fois vécu avec un conjoint } \\
\text { ou un partenaire pendant au moins trois mois? } \\
\text { 4) vous êtes-vous marié pour la première fois? } \\
\text { 5) est né votre (premier) enfant? }\end{array}$ & $\begin{array}{l}\text { 1) d'avoir quitté le foyer parental ? } \\
\text { 2) d'avoir un emploi à temps plein? } \\
\text { 3) de vivre en couple (conjoint/partenaire)? } \\
\text { 4) d'être mère ou père? }\end{array}$ \\
\hline Réponse & $\begin{array}{l}\text { Année, de laquelle on a ensuite retranché l'âge } \\
\text { du répondant (année de l'événement - année de naissance) }\end{array}$ & $\begin{array}{l}\text { Échelle de Likert : } 1 \text { (pas du tout important) } \\
\text { à } 5 \text { (très important) }\end{array}$ \\
\hline
\end{tabular}

(3) Voir également le tableau 1 de l'annexe en fin d'article page 38. 


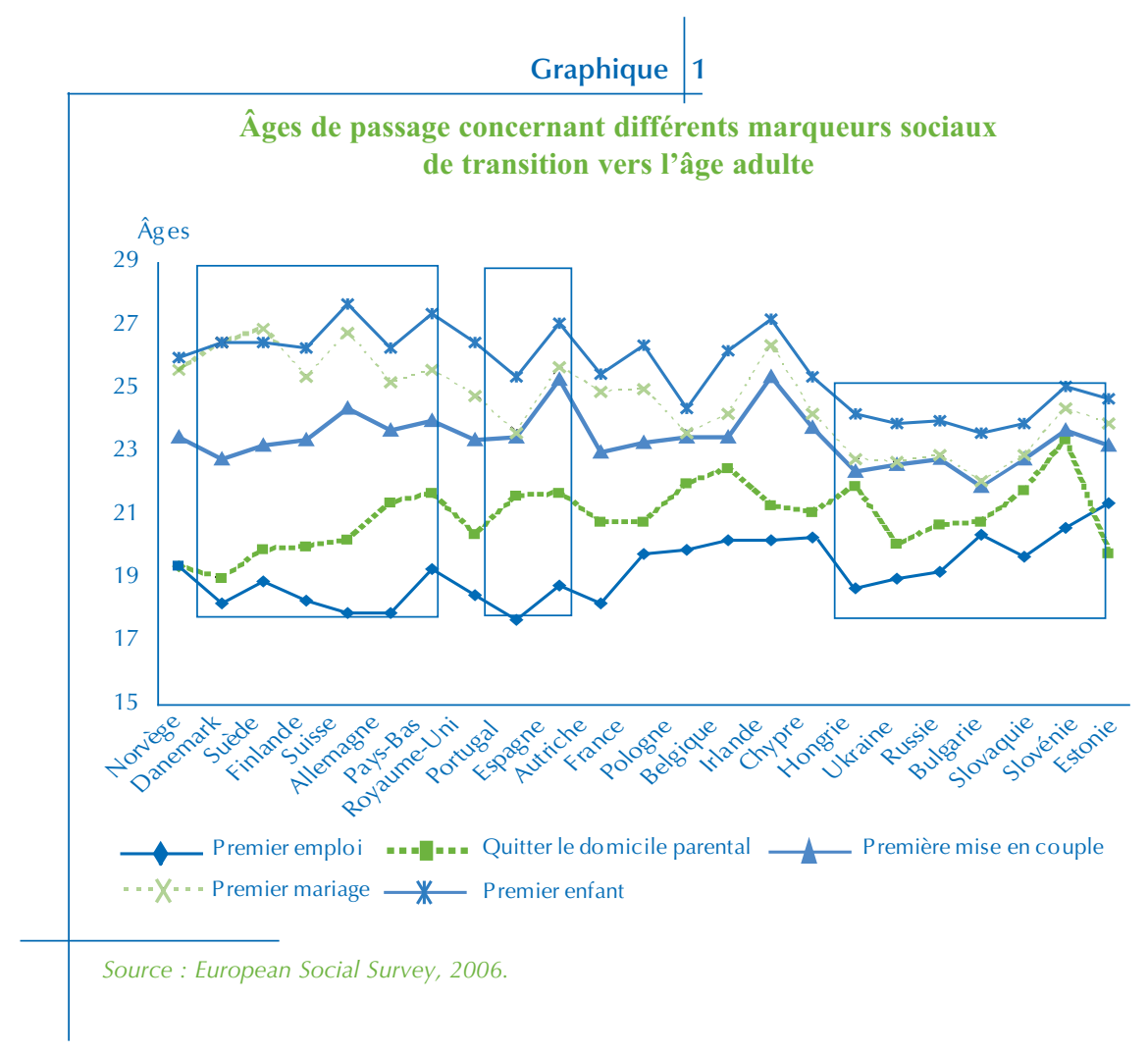

risant des trajectoires scolaires plus longues et débouchant sur davantage de qualifications. $C^{\prime}$ est le cas dans les anciens pays communistes tels que l'Estonie (21,4 ans), la Slovénie (20,6 ans) et la Bulgarie (20,4 ans). Sur l'ensemble des pays participants, la Slovénie et la Bulgarie affichent la plus faible proportion de jeunes (15-29 ans) ayant eu une première expérience professionnelle (respectivement $42,2 \%$ et $47,6 \%$ ) (tableau 2 de I'annexe p. 38).

Une première expérience professionnelle à un âge précoce ne se traduit toutefois pas directement par un départ du foyer parental (graphique 1).

expérience professionnelle a tendance à se produire relativement tôt (17,7 ans en moyenne), c'est-à-dire avant même l'âge de la majorité, tout comme en Suisse et en Allemagne (17,9 ans pour ces deux pays).

Cependant, il est important de noter que le Portugal fait partie des pays dans lesquels la proportion des jeunes âgés de 15 ans à 29 ans ayant eu un emploi est plus faible (48,8\%), contrairement à la Suisse $(73,1 \%)$ et à I'Allemagne $(65,3 \%)$ (tableau 2 de l'annexe p. 38). Ces pays disposent de systèmes d'éducation et de formation très uniformisés et sélectifs et, dans le même temps, leur marché du travail absorbe facilement la main-d'œuvre jeune et peu qualifiée, que ce soit de façon formelle (par exemple via des stages en milieu professionnel et des emplois à temps partiel, en Allemagne et en Suisse) ou informelle (stratégies de sous-emploi ou d'emploi précaire au Portugal) (EGRIS, 2001 ; Walther et al., 2002 a ; Walther et Pohl, 2005). L'existence de ces segments du marché du travail, ainsi que des possibilités éducatives moins flexibles et plus limitées, comptent parmi les facteurs institutionnalisés qui " raccourcissent » la trajectoire scolaire de certains jeunes, essentiellement des garçons issus de milieux sociaux défavorisés.

L'expérience du premier emploi est en revanche plus tardive (après l'âge de 20 ans) dans les pays qui se caractérisent traditionnellement par un système éducatif moins sélectif et plus inclusif, favo-
Le Portugal et I'Allemagne sont les pays dans lesquels l'intervalle qui sépare l'entrée sur le marché du travail et I'autonomie résidentielle est le plus grand: les jeunes quittent le foyer parental en moyenne trois à quatre ans après leur première expérience professionnelle, et après l'âge de 21 ans. En Bulgarie ou à Chypre, en revanche, bien qu'elle se produise plus tard, la première expérience professionnelle est immédiatement suivie du départ du foyer parental, l'intervalle entre les deux événements étant inférieur à un an. Le cas de l'Estonie semble particulier, car il s'agit d'un exemple de séquentialité inverse. L'autonomie résidentielle intervient généralement (en moyenne un an et demi) avant la première expérience professionnelle. Dans les pays scandinaves (Norvège, Danemark et Suède), la première expérience professionnelle et le départ du foyer parental se produisent souvent simultanément (Norvège) ou immédiatement l'un après I'autre. L'autonomie résidentielle intervient plus tôt dans ces pays (aux alentours de 19 ans). Ces pays affichent également le pourcentage le plus élevé de jeunes (15-29 ans) ayant déjà quitté le foyer parental : de $63 \%$ en Suède à $68 \%$ au Danemark (tableau 2 de l'annexe p. 38). Non seulement cette autonomie résidentielle précoce est un schéma culturel d'autonomie des jeunes partagé et apprécié de manière intergénérationnelle, mais elle n'est pas, de plus, étrangère à la politique publique de soutien universel aux jeunes, qu'ils soient étudiants et/ou chômeurs (Walther et al., 2002 b ; Bendit et al., 2004). À l'instar des pays scandinaves, l'Estonie est, encore une fois, le pays d'Europe 
orientale où l'autonomie résidentielle est la plus précoce. L'intervalle qui sépare le départ du foyer parental et la première cohabitation a tendance à être plus long dans ces pays. En Suède et au Danemark, la première expérience de vie en couple est la plus distante du premier mariage, allongeant ainsi les périodes d'expérimentation préconjugale (pour plus de détails, voir le tableau 1 de l'annexe p. 38). De même, ces deux pays sont les pays européens qui comptent le pourcentage le plus élevé de jeunes (15-29 ans) vivant ou ayant vécu en cohabitation (25\% en Suède, 21,4\% au Danemark, 20,1\% en Norvège et $18,5 \%$ en Estonie) (tableau 2 de l'annexe p. 38.). Au Portugal, bien que le délai séparant le départ du foyer parental et la première expérience de vie en couple s'établisse à deux ans en moyenne, l'intervalle entre la première expérience de vie en couple et le premier mariage est faible, ce qui reflète un processus qui correspond toujours au schéma culturel familialiste du mariage, même si les raisons qui le sous-tendent sont d'ordre matériel et économique (Cardoso, 1997). Les expériences de vie en couple sont perçues comme une étape menant au mariage. Ces deux étapes se suivent également de plus près dans les anciens pays communistes, où l'expérimentation de la vie en couple ne constitue pas un schéma récurrent.

On observe les proportions les plus faibles de jeunes (15-29 ans) qui ne vivent pas chez leurs parents au Portugal, en Slovénie, en Pologne et en Bulgarie: moins de $30 \%$ (tableau 2 de l'annexe p. 38) (4). De même, le Portugal est le pays qui affiche le plus faible pourcentage de cohabitation chez les répondants âgés de 15 ans à 29 ans (6\%). La proportion de jeunes mariés, en revanche, est de $12 \%$, les pays d'Europe de l'Est présentant les proportions les plus élevées d'expériences de vie en couple formelles au sein de cette même tranche d'âge : Slovaquie (14,2 \%), Hongrie (15,9\%), Bulgarie $(18,3 \%)$, Pologne (19\%), Russie (20,3\%) et Ukraine $(33,9 \%)$. Contrairement à ce dernier groupe de pays, dans les pays scandinaves, le mariage intervient bien plus longtemps après l'expérience de la vie en couple et tend à être suivi par la formalisation de la vie en couple avec la naissance d'enfant(s), après une longue période de vie commune. En réalité, en Suède, le premier enfant naît, en moyenne, quatre mois avant le premier mariage.
Au Portugal, comme aux Pays-Bas et en Belgique, les couples semblent attendre près de deux ans après le mariage avant de décider de devenir parents (5).

Le graphique 1 fait clairement apparaître la diversité des âges auxquels s'opère la transition vers l'âge adulte en Europe, sur la base des différences d'âge et des intervalles entre les différents marqueurs sociaux : premier emploi, départ du foyer parental, premières expériences de vie en couple, premier mariage et premier enfant. Le modèle le plus courant parmi les divers régimes de transition vers l'âge adulte en Europe présente une séquentialité différée. II se caractérise par une lente transition et par des âges de passage plus tardifs sur le chemin de l'émancipation individuelle, c'està-dire l'émancipation financière et résidentielle (entrée sur le marché du travail et départ du foyer parental). Les marqueurs sociaux qui reflètent une conception plus institutionnalisée de l'installation familiale (premier mariage et premier enfant) interviennent non seulement plus tard, mais souvent après une période prolongée d'expérimentation de la vie en couple. Ce modèle de séquentialité différée est très proche de celui des pays de l'Europe du Nord (Danemark, Finlande, Suède et Norvège), où les périodes d'émancipation individuelle sont relativement courtes et suivies d'une longue période précédant la vie en couple et le mariage. Le contexte institutionnel qui caractérise ces pays produit sans aucun doute des effets sur les variations chronologiques de ces trajectoires. En effet, non seulement la politique sociale de ces pays donne à tous les jeunes des ressources financières suffisantes pour "favoriser» leur autonomie résidentielle, mais le système d'éducation, de formation et d'emploi y est également souple et socialement complet et inclusif (Chambaz, 2001 ; Moreno, 2008; Kugelberg, 1998; Van de Velde, 2005 ; Walther, 2006). La jeunesse tend à être socialement perçue et vécue comme une phase d'exploration (des itinéraires, des situations et des rôles sociaux, etc.) durant laquelle l'autonomie et la réversibilité des options et des décisions ne sont pas critiquées par la société ni perçues négativement mais, au contraire, encouragées culturellement et institutionnellement. Il est possible de faire et de multiplier des expériences diverses et variées, qui ne sont pas perçues comme définitives et irréversibles.

\footnotetext{
(4) Le programme portugais Porta 65 (Porte 65), qui vise à aider les jeunes (âgés de 18 ans à 30 ans) à louer un appartement, en prenant en charge une partie du loyer, illustre bien le décalage entre la politique publique à l'égard des jeunes et la réalité. Même si ce programme public est considéré comme prometteur, voire novateur, son accès est limité non seulement par rapport au nombre de jeunes qui peuvent en bénéficier, mais surtout parce que le marché du logement locatif au Portugal est structurellement très restreint en raison de l'absence d'offres concurrentielles. Ainsi, malgré l'existence de ce programme, la location d'un logement reste très difficile pour la plupart des jeunes dans ce pays.

(5) Bien que le Portugal fasse partie des pays européens qui comptent la plus forte proportion de répondants jeunes ayant des enfants $(12,9 \%)$, immédiatement suivis par les anciens pays communistes tels que la Pologne (15,8 \%), la Hongrie $(17,6 \%)$, la Russie $(21,6 \%)$, la Bulgarie $(26,2 \%)$ et I'Ukraine (26,5\%) (voir le tableau 2 de I'annexe en fin d'article p. 38).
} 
On décide et on choisit en partant du postulat que I'on pourra changer d'avis et opter pour une voie différente à n'importe quel moment et, du point de vue social, cette "expérimentation » est, en quelque sorte, avalisée par la politique sociale de ces pays. Sur ce point, dans les pays au régime de transition universaliste (comme $c^{\prime}$ est le cas des pays d'Europe du Nord), la protection sociale couvre tous les jeunes (âgés de 18 ans et plus), quelle que soit leur situation socio-économique, et l'activité de conseil est largement institutionnalisée à différents stades : scolarité, formation et emploi. La conception de la jeunesse privilégie le développement personnel et la capacité des jeunes à choisir, définir et redéfinir leurs choix de vie et de trajectoires (Walther, 2006).

Dans les pays d'Europe centrale, où les régimes de transition sont marqués par un contexte institutionnel et social moins généreux envers les jeunes, comme la Suisse (où l'intervalle entre deux marqueurs sociaux est plus long), I'Allemagne, les Pays-Bas, l'Autriche, le Royaume-Uni ou l'Irlande, les phases d'émancipation individuelle sont plus longues et le délai entre l'entrée sur le marché du travail et le départ du foyer parental également. Bien que les régimes de transition de la jeunesse soient très différents dans certains pays, ils ont ceci de commun que les jeunes sont confrontés à des conditions sociosymboliques méritocratiques qui les encouragent à valoriser le processus d'acquisition d'une indépendance financière et vis-à-vis des parents à un stade relativement précoce. Cependant, bien souvent, la réalité sociale ne facilite pas ce processus, surtout en ce qui concerne l'entrée sur le marché du travail. Le Portugal, et surtout l'Espagne, se caractérisent par une longue période de transition vers l'âge adulte, même si l'expérimentation de la vie en couple y est relativement absente, la première expérience de vie à deux et le premier mariage étant très proches dans le temps. Les contextes institutionnels de ces pays témoignent d'un régime de transition sousprotecteur, avec une politique publique sommaire sur ce plan, qui reporte sur les familles une grande part des responsabilités, lesquelles sont donc tributaires des contingences sociales propres aux familles, ainsi que du caractère traditionnellement restrictif et précaire du système d'emploi. Par conséquent, les jeunes restent plus longtemps chez leurs parents, quand bien même ils sont (sous-)intégrés au marché du travail, et jusqu'à ce qu'ils fondent une nouvelle famille dans laquelle les responsabilités financières peuvent être partagées.

Les périodes typiques des transitions vers l'âge adulte au sein des pays d'Europe de l'Est se caractérisent par un profil de séquentialité contiguë, soit un rythme de transition rapide et l'enchaînement des marqueurs de passage à intervalles rapprochés.
Dans ces pays, devenir adulte résulte non pas d'une logique d'expérimentation et d'émancipation individuelle, mais de l'urgence de "fonder » une famille, avec un mariage précoce et l'arrivée d'enfants peu après. Ce profil met en évidence un modèle de transition plus traditionnel, linéaire et standardisé, transition perçue essentiellement comme une période de préparation à une intégration définitive dans les rôles familial et professionnel qui définissent la transformation d'un jeune en « adulte », laquelle est appréhendée comme une ascension sociale. Ce profil concerne des pays tels que la Bulgarie, la Slovénie, la Slovaquie, la Russie, I'Ukraine, la Pologne et la Hongrie.

\section{Évaluation des marqueurs sociaux associés au passage à la vie d'adulte}

Dans les sociétés européennes contemporaines, la déstandardisation et l'indéfinition chronologique du passage à la vie adulte pourraient ajouter à la difficulté d'établir des formes normatives et prescriptives de cette transition. Certains des rituels traditionnels célébrant ce passage ne sont plus des institutions immuables et incontestables. Atteindre les marqueurs sociaux traditionnels est une question de volonté : ils ne sont plus poursuivis par tous et ces événements perdent en synchronie dans le temps et dans l'espace social. Dans le même ordre d'idées, est-ce que les marqueurs sociaux, tels que le départ du foyer parental, I'obtention d'un emploi à plein temps, la vie en couple ou la parentalité, perdent en importance dans la notion de «devenir adulte »? Quelle importance les marqueurs traditionnels de passage conservent-ils dans cette définition? Lorsque l'on pose la question de l'importance accordée à chacun de ces marqueurs sociaux de passage à " l'âge adulte », on note différentes attitudes (tableau 3), qui peuvent correspondre à différents schémas de (dé)ritualisation, présentés dans la suite de l'article.

L'autonomie résidentielle (départ du foyer parental) constitue le marqueur social le plus important dans les pays où ce processus de transition s'opère également le plus tôt, c'est-à-dire parmi les pays nordiques (Finlande, Suède, Norvège et Danemark). L'Espagne fait figure d'exception: bien qu'intervenant plus tard, le départ du foyer parental est perçu comme un marqueur très important vers la vie d'adulte. Cependant, nous pensons que la valeur symbolique attachée à I'autonomie résidentielle en Espagne diffère de celle sous-jacente en Europe du Nord. Dans les pays d'Europe du Nord, quitter le foyer parental représente le début d'un long processus de transition vers I'«âge adulte », caractérisé par l'expérimentation, l'hédonisme et la découverte de soi-même (Kugelberg, 1998), et par des mouvements d'aller-et-retour 


\section{\begin{tabular}{l|l} 
Tableau & 3
\end{tabular}}

Hiérarchisation des marqueurs sociaux dans la transition vers I'«âge adulte» (moyennes)

\begin{tabular}{|c|c|c|c|c|}
\hline $\begin{array}{c}\text { Niveau } \\
\text { d'importance }\end{array}$ & $1^{\mathrm{er}}$ & $2^{\mathrm{e}}$ & $3^{e}$ & $4^{e}$ \\
\hline Finlande & Logement $(3,17)$ & Emploi $(3,07)$ & Vie en couple $(2,42)$ & Enfants $(2,07)$ \\
\hline Suède & Logement $(3,03)$ & Emploi $(2,48)$ & $\begin{array}{l}\text { Vie en couple }(1,90) \\
\text { Enfants }(1,90)\end{array}$ & - \\
\hline Norvège & Logement $(3,11)$ & Emploi $(2,78)$ & $\begin{array}{l}\text { Vie en couple }(2,08) \\
\text { Enfants }(2,11)\end{array}$ & - \\
\hline Danemark & Logement $(3,49)$ & $\begin{array}{l}\text { Emploi }(2,96) \\
\text { Enfants }(3,03)\end{array}$ & Vie en couple $(2,53)$ & - \\
\hline Espagne & Logement $(2,34)$ & $\begin{array}{l}\text { Emploi }(2,57) \\
\text { Enfants }(2,32)\end{array}$ & Vie en couple $(2,15)$ & - \\
\hline Suisse & $\begin{array}{l}\text { Logement }(2,92) \\
\text { Emploi }(2,88)\end{array}$ & Enfants $(2,67)$ & Vie en couple $(2,41)$ & - \\
\hline Pays-Bas & $\begin{array}{l}\text { Logement }(2,64) \\
\text { Emploi }(2,63)\end{array}$ & $\begin{array}{l}\text { Enfants }(2,28) \\
\text { Vie en couple }(2,25)\end{array}$ & - & - \\
\hline Autriche & Emploi $(3,39)$ & Logement $(3,11)$ & Vie en couple $(2,59)$ & Enfants $(2,46)$ \\
\hline Royaume-Uni & Emploi $(3,12)$ & Logement $(2,68)$ & $\begin{array}{l}\text { Vie en couple }(2,28) \\
\text { Enfants }(2,32)\end{array}$ & - \\
\hline Irlande & Emploi $(3,16)$ & Logement $(2,58)$ & $\begin{array}{l}\text { Vie en couple }(2,23) \\
\text { Enfants }(2,21)\end{array}$ & - \\
\hline Belgique & Emploi $(3,21)$ & $\begin{array}{l}\text { Logement }(2,62) \\
\text { Enfants }(2,57)\end{array}$ & Vie en couple $(2,42)$ & - \\
\hline France & Emploi $(3,60)$ & $\begin{array}{l}\text { Logement }(2,97) \\
\text { Enfants }(3,01)\end{array}$ & Vie en couple $(2,61)$ & - \\
\hline Estonie & Emploi $(3,35)$ & $\begin{array}{l}\text { Logement }(2,97) \\
\text { Enfants }(2,89)\end{array}$ & Vie en couple $(2,78)$ & - \\
\hline Chypre & Emploi $(3,38)$ & Enfants $(3,13)$ & Vie en couple $(2,89)$ & Logement $(2,68)$ \\
\hline Slovaquie & Emploi $(3,21)$ & $\begin{array}{l}\text { Logement }(2,38) \\
\text { Vie en couple }(2,44)\end{array}$ & $\begin{array}{l}\text { Enfants }(2,49) \\
\text { Vie en couple }(2,44)\end{array}$ & - \\
\hline Hongrie & Emploi $(2,65)$ & $\begin{array}{l}\text { Logement }(2,10) \\
\text { Vie en couple }(2,07) \\
\text { Enfants }(2,06)\end{array}$ & - & - \\
\hline Slovénie & Emploi $(3,14)$ & $\begin{array}{l}\text { Vie en couple }(2,57) \\
\text { Enfants }(2,64)\end{array}$ & $\begin{array}{l}\text { Logement }(2,70) \\
\text { Enfants }(2,64)\end{array}$ & - \\
\hline Allemagne & $\begin{array}{l}\text { Emploi }(2,88) \\
\text { Enfants }(2,81)\end{array}$ & $\begin{array}{l}\text { Logement }(2,75) \\
\text { Enfants }(2,81)\end{array}$ & Vie en couple $(2,59)$ & - \\
\hline Pologne & $\begin{array}{l}\text { Emploi }(3,17) \\
\text { Enfants }(3,16)\end{array}$ & Vie en couple $(2,98)$ & Logement $(2,63)$ & - \\
\hline Bulgarie & Enfants $(3,80)$ & Emploi $(3,77)$ & Vie en couple $(3,48)$ & Logement $(2,67)$ \\
\hline Portugal & Enfants $(3,56)$ & $\begin{array}{l}\text { Emploi }(3,38) \\
\text { Vie en couple }(3,34)\end{array}$ & Logement $(3,01)$ & - \\
\hline Ukraine & Enfants $(3,79)$ & $\begin{array}{l}\text { Emploi }(3,43) \\
\text { Vie en couple }(3,47)\end{array}$ & Logement $(3,17)$ & - \\
\hline Russie & Enfants $(3,64)$ & Vie en couple $(3,38)$ & Emploi $(3,28)$ & Logement $(3,16)$ \\
\hline \multicolumn{5}{|c|}{$\begin{array}{l}\text { Source : European Social Survey, } 2006 . \\
\text { Échelle de } 1 \text { (pas du tout important) à } 5 \text { (très important). Comparaisons par paires au moyen du test de Bonferroni, avec } p<0,01 . \\
\text { Logement : départ du foyer parental ; emploi : exercice d'un emploi à plein temps; vie en couple : vivre avec un conjoint ou un } \\
\text { concubin ; enfants : devenir parent. } \\
\text { Lorsque plusieurs catégories apparaissent dans la même cellule, c'est qu'elles ne présentent pas un écart statistiquement significatif. } \\
\text { Ce tableau montre la hiérarchisation des marqueurs sociaux selon l'importance attribuée par les individus de chaque pays. Les résultats } \\
\text { sont organisés en ordre décroissant d'importance, et les chiffres entre parenthèses indiquent le degré d'importance associé à chaque } \\
\text { marqueur social. }\end{array}$} \\
\hline
\end{tabular}


(Pais, 1996 ; Du Bois-Reymond et López Basco, 2003). Dans le cas de I'Espagne, en revanche, I'autonomie résidentielle représente un marqueur social fondamental et déterminant dans l'accès à la vie adulte, et I'affirmation définitive que le jeune a atteint les conditions nécessaires à son émancipation sociale et individuelle, à savoir la stabilité financière et la volonté d'assumer des responsabilités familiales.

En d'autres termes, la raison pour laquelle ce marqueur social est jugé si important découle sans doute directement du fait qu'il est si difficile à atteindre dans les pays d'Europe du Sud. En effet, ces pays se caractérisent par des opportunités $d^{\prime}$ emploi et d'indépendance économique très réduites, par un modèle culturel familialiste où la norme consiste à quitter le foyer parental pour fonder une nouvelle famille, et par l'absence de politique publique favorisant l'autonomie résidentielle grâce à des niveaux de prix du logement accessibles compte tenu du niveau de vie moyen et des ressources financières des jeunes Espagnols (Moreno, 2008 ; Van de Velde, 2005). Après I'autonomie résidentielle, l'exercice d'un emploi à plein temps est le deuxième marqueur social le plus important pour le passage à l'âge adulte dans les pays d'Europe du Nord, ce qui montre que les marqueurs d'émancipation individuelle sont davantage valorisés que les marqueurs orientés sur la famille, comme le fait de vivre en couple ou de devenir parents. Ce constat vaut également en Suisse et aux Pays-Bas, où I'on accorde la même valeur au départ du foyer parental et à l'exercice $d^{\prime}$ un emploi à plein temps.

La première et la deuxième place accordées respectivement à la valorisation symbolique des marqueurs d'émancipation individuelle et à l'installation familiale s'observent également dans des pays comme l'Autriche, le Royaume-Uni, I'Irlande et I'Estonie même si, dans ces pays, un emploi à plein temps est perçu comme plus important que l'autonomie résidentielle, probablement parce qu'il est considéré comme une condition préalable à l'obtention du second. Contrairement à ce qui se passe en Europe du Nord, dans ces pays, le système d'État-providence ne favorise pas l'autonomie résidentielle des jeunes, laquelle dépend uniquement de leurs revenus (Chambaz, 2001 ; Cicchelli, 2001). Les jeunes sont censés faire leur chemin, seuls, sans dépendre de leurs parents ou de l'État pour accomplir leur passage à l'âge adulte. Dans ces pays, l'éthique du travail et I'autonomie financière sont particulièrement valorisées dans les trajectoires des jeunes. En outre, on notera avec intérêt que, pour certains pays, devenir parent est perçu comme hiérarchiquement plus important que vivre en couple, par exemple en Estonie. Cela s'explique vraisemblablement par l'irréversibilité, et donc la responsabilité sociale, associée à ce marqueur. II n'en va pas de même pour la vie en couple, puisque I'on peut facilement se séparer ou divorcer.

La logique de l'installation familiale s'observe dans le groupe de pays dans lesquels ce modèle de transition vers le statut d'adulte se matérialise également par des pratiques concrètes. C'est le cas du Portugal et de certains anciens pays communistes (Pologne, Bulgarie, Ukraine et Russie), où l'autonomie résidentielle finit par être un marqueur social sous-valorisé, sauf si elle est associée à la fondation d'une nouvelle famille. Même parmi les pays qui accordent la première place à l'emploi à plein temps (Slovaquie, Slovénie ou Hongrie), le classement en deuxième position de la parentalité et de la vie en couple reflète l'importance symbolique que revêt la fondation d'une famille avec des enfants pour être considéré comme " adulte», ce qui n'est possible qu'après s'être assuré une source de revenus stable et régulière.

\section{Profils des attitudes vis-à-vis de la transition à la vie adulte}

Même si les attitudes des individus s'inscrivent dans le contexte du pays dans lequel ils vivent, leurs schémas normatifs peuvent également être étendus à la coexistence de différents positionnements en terme d'attitude par rapport aux marqueurs sociaux perçus comme importants dans la transition à l'âge adulte. Dans cet esprit, on a privilégié, dans notre stratégie analytique, les grappes d'individus (10) plutôt que les habituelles grappes de pays, afin de se faire une idée plus précise sur cette hypothèse. Ainsi, une analyse par grappes en deux temps a été menée, en englobant les répondants des vingt-trois pays et en prenant en compte les variables sur le niveau d'importance attribué aux marqueurs sociaux associés à I'entrée dans l'âge adulte. Les résultats mettent en évidence quatre grappes différentes qui rendent compte d'une pertinence à la fois statistique et sociologique concernant le processus de déritualisation à travers I'Europe (tableau 4). La définition et la classification des grappes reposent sur les réponses (moyennes) apportées par les répondants (dans chaque grappe) aux variables incluses dans I'analyse. L'analyse en grappes repose sur les schémas

(10) Pour l'analyse en grappes en deux temps, on a utilisé l'échantillon total. Ainsi, chacun des répondants a été inclus dans l'une de ces grappes, quel que soit son pays d'origine. L'analyse qui suit montrera comment les différents profils d'attitude sont, chacun, distribués au sein de chaque pays européen (graphique 2 p. 32). 


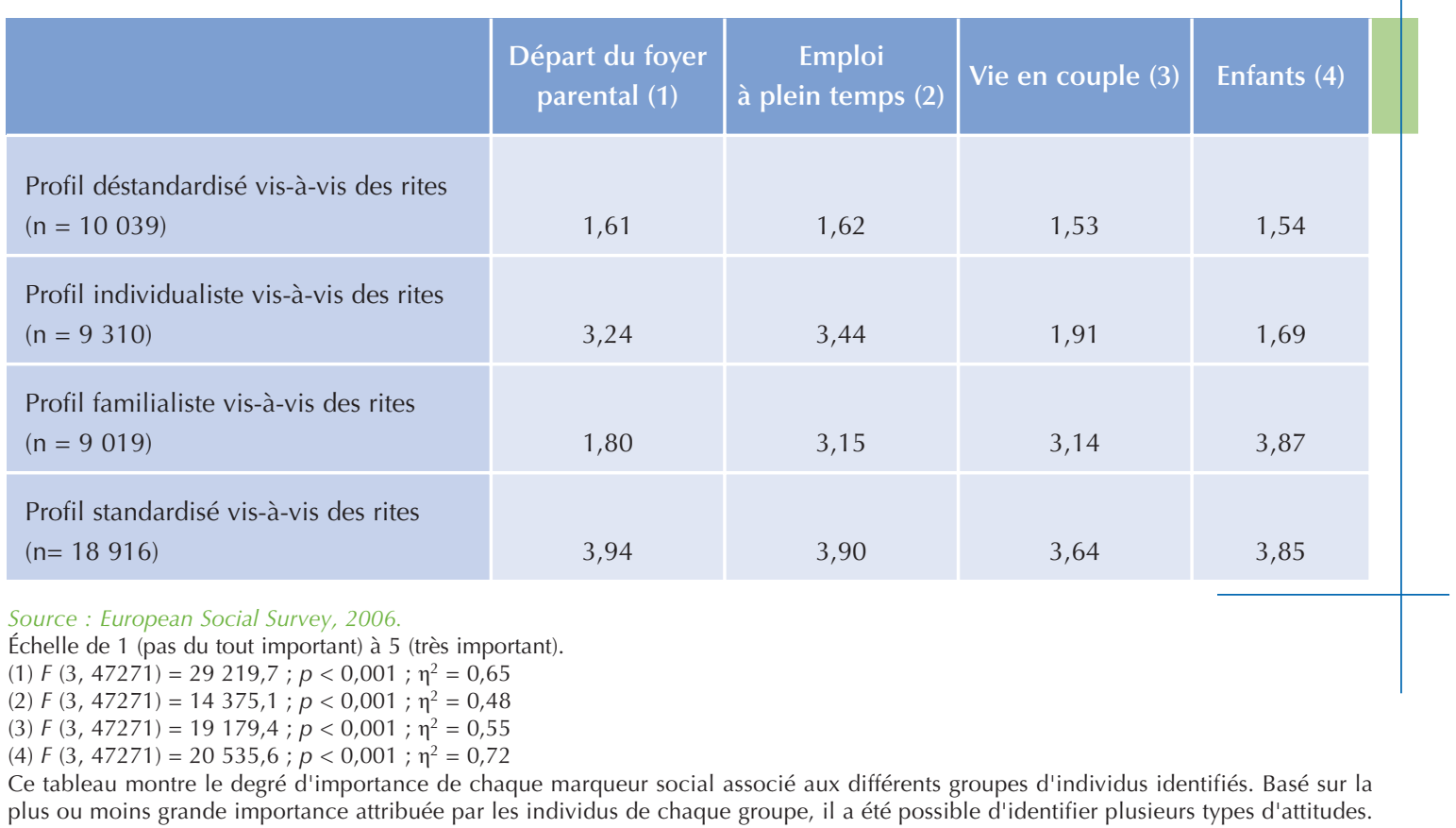

de proximité contenus dans un jeu de données, autrement dit, sur la prise en compte des similarités et des différences parmi les profils de réponses des répondants pour un jeu donné de variables : les répondants ayant un profil de réponse analogue sont regroupés dans la même grappe.

La première grappe rassemble des individus qui présentent une attitude davantage déstandardisée vis-à-vis des rites du processus social qui permet de devenir adulte, car ils ne perçoivent aucun de ces marqueurs sociaux comme importants dans le contexte de cette transition. Les personnes classées dans cette grappe ne se conforment pas à une lecture normative des événements et des étapes qui définissent la transition vers l'âge adulte : elles amoindrissent la valeur sociale normative de ces marqueurs et, très vraisemblablement, accordent davantage d'importance aux marqueurs biographiques et individuels ancrés dans l'histoire personnelle de l'individu. Les attitudes déstandardisées vis-à-vis des rites peuvent être un indicateur, ou une conséquence, d'une fusion des événements, ainsi que de leur enchaînement moins linéaire et de leur réversibilité grandissante. Les marqueurs sociaux traditionnels sont perçus comme moins saillants et plus flous dans la définition de cette transition ; devenir adulte est plus étroitement lié aux expériences subjectives de développement personnel de chacun (11). La deuxième grappe correspond à des individus qui font preuve d'une attitude individualiste vis-à-vis des rites de passage à l'âge adulte car pour eux, les marqueurs sociaux liés à l'émancipation individuelle, et plus précisément à l'indépendance économique et résidentielle, revêtent une importance plus grande dans I'accomplissement de cette transition. La troisième grappe, pour sa part, dénote davantage une attitude familialiste vis-à-vis des rites. En effet, les répondants de ce groupe accordent davantage d'importance aux marqueurs sociaux liés aux événements familiaux et à la fondation d'une nouvelle cellule familiale : vivre en couple et devenir parent. Dans ce contexte, être financièrement autonome ne revêt pas la même signification qu'être économiquement indépendant: il s'agit d'être capable de pourvoir aux besoins de sa famille. La quatrième et dernière grappe regroupe des personnes qui présentent plutôt une attitude standardisée vis-à-vis des rites. Tous les marqueurs sociaux sont perçus comme importants dans la transition à l'âge adulte. Ainsi, ce passage est vu selon une perspective normative et régulée par une séquence d'événements. Devenir adulte est lié à l'accomplissement d'une trajectoire standard, institutionnalisée et répondant à des règles.

(11) Par exemple, ils privilégient la responsabilité personnelle et le sens des responsabilités, la possibilité de prendre ses propres décisions ou une relation aux parents reposant sur l'égalité. Au sujet de l'opérationnalisation de ces critères d'épanouissement personnel, entre autres, concernant l'idée que se font les jeunes de l'âge adulte, voir Arnett (1997) (États-Unis) et Plug et al. (2003) (Europe). 
Les grappes d'attitudes identifiées suivent une distribution variable selon les pays analysés. La proportion d'individus affichant une attitude déstandardisée vis-à-vis des rites de passage est plus forte dans les pays tels que l'Espagne, la Hongrie et les Pays-Bas. Au sein de ce groupe de pays, la proportion affichant une attitude individualiste vis-à-vis des rites est également élevée, de même que dans les cas de la Suède, de I'Irlande, du Royaume-Uni, de la Norvège, de la Finlande et de la Belgique (graphique 2). Pour leur part, la Pologne, I'Ukraine, la Russie et la Bulgarie sont les pays où la proportion d'attitudes standardisées vis-à-vis des rites de passage est la plus forte, les attitudes familialistes étant également largement représentées dans ces pays, tout comme en Pologne et en Allemagne.

Si on met de côté le contexte institutionnel qui caractérise chaque pays, dans quelle mesure les caractéristiques individuelles façonnent-elles ces différents positionnements? Afin de se faire une idée plus précise et d'étendre le champ de l'analyse aux caractéristiques individuelles associées aux différents profils d'attitudes identifiés, une analyse des correspondances multiples a été réalisée sur les variables suivantes: les quatre grappes, le genre, la classe d'âge, le niveau d'études et la pra- tique religieuse. La variable "pays » a également été incluse comme variable supplémentaire ; cette variable n'est pas prise en compte pour la définition des dimensions sous-jacentes mais elle permet de situer la position d'un pays sur une représentation graphique pluridimensionnelle (12). Les valeurs d'inertie indiquent la pertinence de deux dimensions analytiques. Les mesures de discrimination montrent que la première dimension s'intéresse aux variables qui correspondent à des conditions de vie sociosymboliques différentes et contrastées, alors que la seconde dimension est représentée par les différentes attitudes vis-à-vis des rites identifiées pour la transition vers l'âge adulte.

Le graphique pluridimensionnel (graphique 3) met en évidence les tendances qui sont apparues dans les analyses précédentes, ainsi que la manière dont ces différentes grappes correspondent à des attitudes opposées qui, de la même façon, se rapprochent des différentes caractéristiques sociosymboliques des individus. Il est intéressant de noter que les différents profils d'attitudes apparaissent chacun dans une zone différente du graphique, corroborant l'idée qu'ils correspondent à des attitudes plurielles et contrastées visà-vis de la transition vers l'âge adulte. D'un point

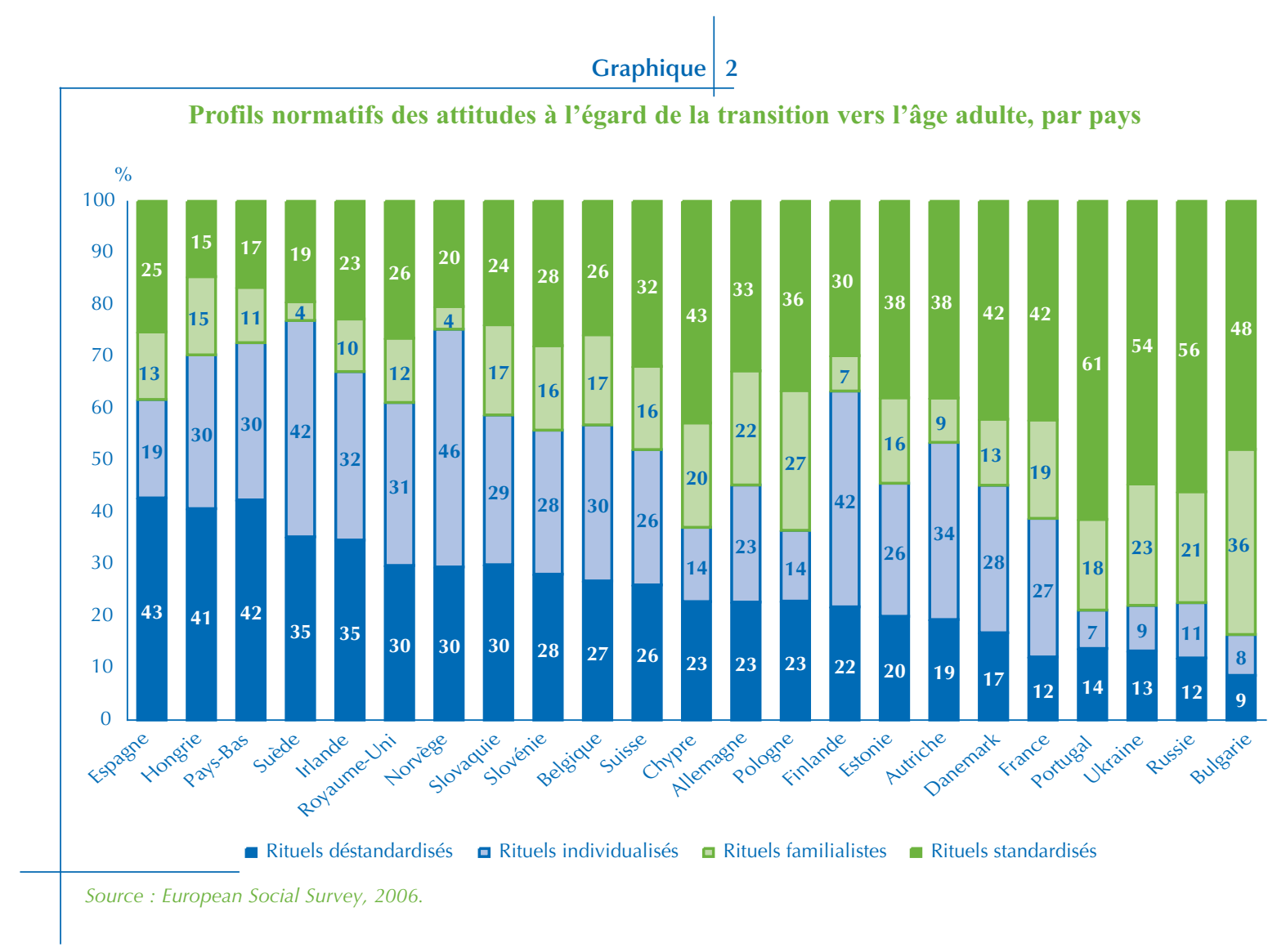

(12) La méthode de normalisation de l'objet principal (objet principal normalisation) a été retenue car elle maximise les dimensions de la distance et de l'opposition entre les différents objets cartographiés. 


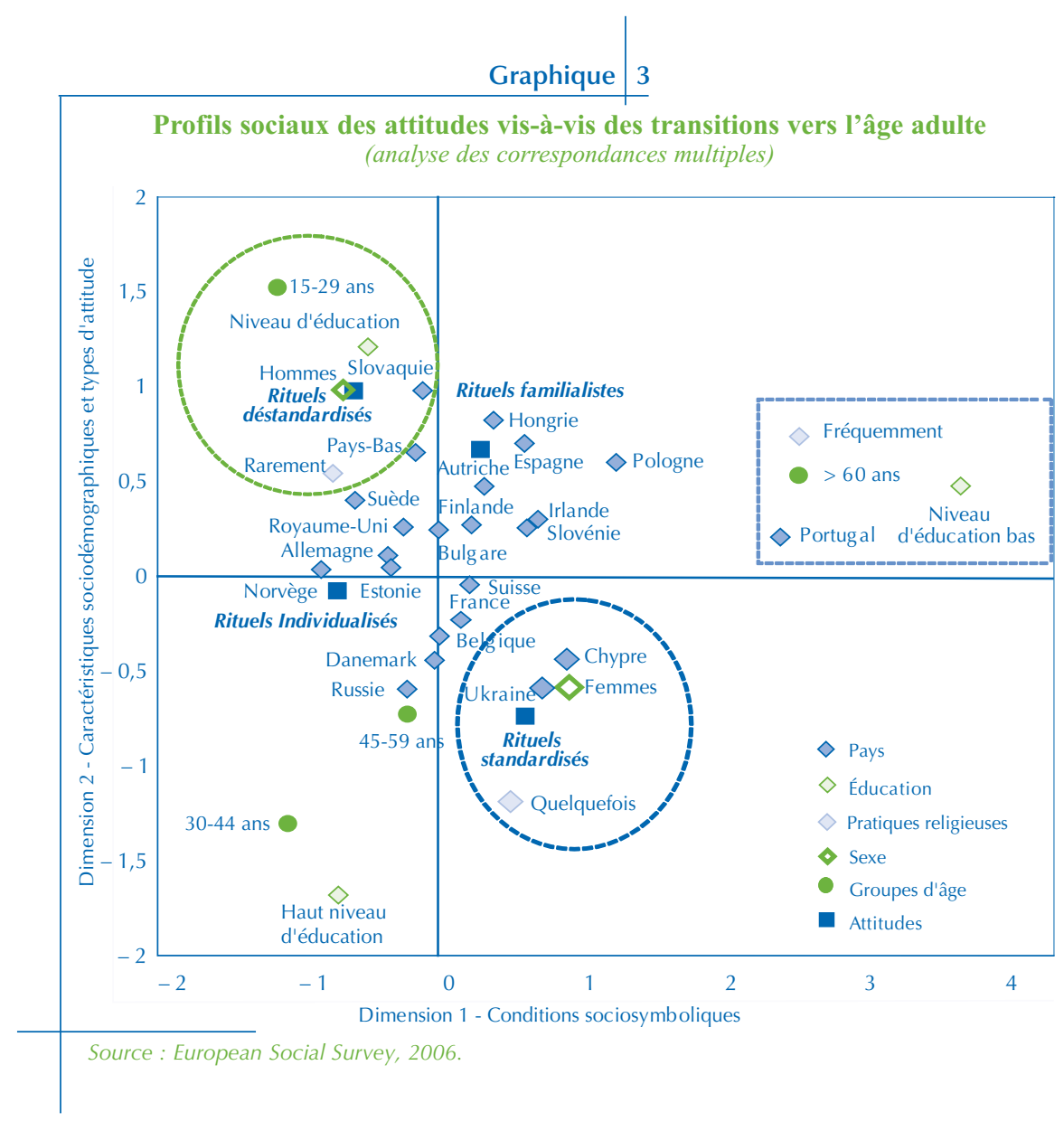

fréquemment à des offices religieux. À proximité de ce profil, figurent également la Pologne, la Hongrie, I'Espagne, I'Irlande et la Slovénie. Bien que certaines attitudes se rapprochent davantage de certains contextes nationaux, cette analyse signale que des individus aux caractéristiques sociosymboliques et sociodémographiques différentes présentent des profils de positionnement distincts vis-àvis du passage à la vie adulte. De manière générale, les individus plus jeunes, plus instruits et moins religieux ont tendance à faire preuve d'une attitude plus déstandardisée et individualiste, alors que les personnes plus âgées, qui ont fait moins d'études et sont plus religieuses, se rapprochent des atti-

de vue conceptuel, ces profils d'attitudes peuvent être traités comme des pôles contrastés où les attitudes individuelles, associées à différentes conditions sociosymboliques et à différents pays d'Europe, se rapprochent plus ou moins d'un pôle ou de l'autre. Les attitudes déstandardisées vis-àvis des rites sont associées à des hommes jeunes (âgés de 15 ans à 29 ans) ayant un niveau d'études moyen, et qui assistent rarement à des offices religieux. Les pays les plus proches de ce profil sont la Slovaquie, les Pays-Bas, la Suède et le Royaume-Uni. À l'opposé, on peut observer que les attitudes standardisées vis-à-vis des rites sont associées à des femmes qui assistent à des offices religieux. Chypre et Ukraine sont les pays qui se rapprochent le plus de ce profil.

À proximité de ces profils, et également dans des zones opposées du graphique, on observe des positionnements qui reflètent les attitudes individualistes et familialistes vis-à-vis des rites du passage à la vie adulte. Le profil individualiste semble associé à des individus affichant un niveau d'études élevé, âgés de 30 ans à 59 ans. Les pays qui se rapprochent le plus de ce profil sont le Danemark et I'Estonie. Le Portugal se trouve dans le pôle familialiste, mais proche du pôle standardisé ; il est associé à des individus ayant un niveau d'études faible, âgés de 60 ans et plus, et qui assistent tudes familialistes et standardisées. Le sexe semble également être une source de différenciation : les femmes sont plus proches d'une perspective orientée sur la famille que les hommes. En conséquence, même si les contextes normatifs et institutionnels contribuent à façonner les attitudes individuelles, les rites sont également perçus et valorisés différemment par les individus en fonction de leurs caractéristiques sociales et culturelles. Le même cadre institutionnel peut être intégré et, in fine, vécu de façon très différente par les individus. Globalement, des profils d'attitudes variés vis-à-vis du passage au statut d'adulte (co)existent à l'intérieur des pays européens et d'un pays à l'autre, et ces profils sont, dans le même temps, influencés et façonnés par le contexte national et socioculturel.

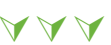

\section{Conclusion}

Alors même que la jeunesse a tendance à se prolonger, la transition à l'âge adulte acquiert de nouvelles caractéristiques : elle est retardée et devient plus complexe. Cependant, ce processus ne revêt pas la même importance ni les mêmes caractéristiques dans tous les pays d'Europe. Sur le plan de 
la déstandardisation, il semble exister, à travers le continent, un schéma chronologique dominant en ce qui concerne les marqueurs de passage, et une réorganisation différente de l'ordre traditionnel des événements n'a été constatée que dans quelques pays, tels que l'Estonie, où l'on a tendance à quitter le foyer parental avant d'entrer sur le marché du travail, ou encore dans les pays du Nord tels que la Suède ou le Danemark, où I'on devient père ou mère avant ou en même temps que I'on se marie. Même si aucune discontinuité majeure n'a été observée, les périodes associées aux marqueurs du passage au statut d'adulte varient. On s'en rend clairement compte lorsque l'on examine les événements et la durée des séquences (intervalles). Même si un schéma de séquentialité différée est très présent, la période correspondant au processus de transition vers l'âge adulte en Europe semble plus rapide parmi les anciens pays communistes, tels que la Bulgarie, la Russie, I'Ukraine, la Pologne et la Slovaquie. Au sein de ce groupe de pays, les marqueurs traditionnels tendent à s'enchaîner rapidement. La jeunesse est vécue comme une phase de "formation » aux rôles associés à l'âge adulte, reflétant un modèle de transition statutaire où les marqueurs se suivent rapidement.

Par ailleurs, parmi les pays d'Europe du Nord, le processus de transition vers l'âge adulte est davantage prolongé, commençant tôt et finissant tard, avec des intervalles plus longs entre l'émancipation individuelle et les événements marquant l'installation familiale. Dans les pays comme la Norvège, le Danemark, la Suède ou la Finlande, l'entrée sur le marché du travail et le départ du foyer parental interviennent tôt et se suivent de près. On observe, en revanche, que les marqueurs plus familialistes, comme le mariage ou le fait d'avoir des enfants, arrivent plus tard. Entre l'émancipation individuelle et la fondation d'une nouvelle famille, les jeunes ont le temps de tester et d'explorer différentes situations résidentielles, vocationnelles, professionnelles et de vie à deux, ainsi que d'autres expériences biographiques. En conséquence, les conditions institutionnelles du processus de déstandardisation dans le contexte de ces pays peuvent se traduire par une déritualisation du passage à l'âge adulte, celui-ci devenant moins conventionnel et moins institutionnalisé, et davantage individualisé et associé à des tournants biographiques.

Des pays tels que le Portugal et l'Espagne affichent des périodes plus longues de transition vers l'état d'adulte, mais avec des séquences différentes par rapport aux modèles identifiés précédemment. Après le premier emploi à temps plein, que I'on prend généralement plus tôt que dans les pays du nord de l'Europe, les jeunes restent pendant une période prolongée chez leurs parents. En d'autres termes, même si le processus d'acquisition de
I'autonomie financière se produit tôt, il n'est pas immédiatement suivi de l'autonomie résidentielle en raison des facteurs économiques, institutionnels et aussi culturels évoqués. Au Portugal, le départ du foyer parental a tendance à être suivi par la fondation d'une nouvelle famille (se marier et/ou avoir des enfants), alors qu'en Espagne, ces marqueurs interviennent plus tard dans la vie. En conséquence, dans le cas de l'Espagne, la transition à la vie adulte constitue in fine une période plus longue, propice à l'expérimentation, caractéristique de trajectoires de jeunesse moins standardisées et plus flexibles. Cette situation n'est pas si fréquemment observée au Portugal.

Le modèle de séquentialité identifié en ce qui concerne les expériences vécues est analogue aux profils d'attitudes valoratifs relatifs aux marqueurs traditionnels de passage à l'âge adulte. L'analyse du processus de déritualisation a permis, en réalité, de comprendre la valeur symbolique attribuée à un modèle d'émancipation individuelle parmi les répondants des pays d'Europe du Nord (qui privilégient l'autonomie résidentielle et, en second lieu, l'emploi à plein temps), alors que les pays postcommunistes ont toujours tendance à valoriser le modèle de l'installation familiale (avoir des enfants et vivre en couple sont les marqueurs perçus comme les plus importants pour la transition à l'âge adulte).

De façon analogue à ce qui a été constaté pour les expériences entre les différents marqueurs de passage, les positionnements en termes d'attitudes au Portugal et en Espagne ne reflètent pas I'homogénéité que des expressions comme " pays d'Europe du Sud " ou " pays méditerranéens » pourraient laisser supposer. Au contraire, les résultats tendent vers un positionnement différent en termes d'attitudes entre ces deux pays en ce qui concerne la valeur symbolique des marqueurs de passage. Même si le Portugal et I'Espagne présentent des séquences de transition prolongées et structurellement analogues (bien que les marqueurs interviennent à des moments différents), du point de vue symbolique, I'Espagne tend à être plus proche du modèle d'émancipation individuelle, percevant l'autonomie résidentielle comme le marqueur le plus important vers la vie adulte. En revanche, le Portugal tend à être plus proche d'un modèle plus traditionnel et plus institutionnalisé d'installation familiale non seulement en termes d'expériences mais aussi sur le plan symbolique. Même si ces deux pays se caractérisent par des institutions sous-protectrices en ce qui concerne les transitions des jeunes, le Portugal présente la plus forte proportion $(61 \%$ ) de répondants qui affichent une attitude plus standardisée vis-à-vis des rites, tous les marqueurs de passage étant jugés importants, alors qu'en Espagne, la plus forte proportion 
de répondants (43\%) affiche une attitude déstandardisée vis-à-vis des rites, car tous les marqueurs de passage y sont perçus comme peu importants dans l'entrée dans l'âge adulte. Cette situation reflète une vision plus détachée de la perspective traditionnelle, linéaire et normative de transition vers l'âge adulte dans le cas de l'Espagne.

En outre, ces modèles normatifs correspondent à des pôles opposés et sont associés à des profils sociaux différents. Des attitudes déstandardisées vis-à-vis des rites sont associées à des groupes plus jeunes, à des niveaux d'études moyens et à une faible pratique religieuse, et sont proches de positionnements plus individualistes. En revanche, les attitudes plus standardisées vis-à-vis des rites sont associées à des profils socioculturels plus conservateurs, c'est-à-dire à des groupes plus âgés, au niveau d'études plus faible et plus proches de positionnements familialistes. Si on se penche sur la distribution des pays au sein des différents pôles d'attitudes relatives à la transition vers I'âge adulte, on perçoit clairement une forte proportion d'attitudes familialistes et standardisées vis-à-vis des rites de passage au Portugal. Alors que, pour certains autres pays, le positionnement n'est pas si marqué, les principales tendances identifiées sont corroborées et associées au profil socioculturel. Cela signifie que les conditions institutionnelles des pays, même si elles constituent une variable limitative importante, ne sont pas les seuls déterminants de la diversité des processus de transition vers l'état d'adulte à travers l'Europe. Des variables structurelles dans le contexte de chaque pays interviennent également dans leur complexification et leur diversification, montrant que différentes (ré)actions sociales peuvent s'ancrer dans le même contexte national et institutionnel. Ainsi, la pluralisation des processus de déstandardisation et de déritualisation s'observe à la fois dans les pays européens et entre les différents pays.

\section{\begin{tabular}{l|l} 
& \\
Références & bibliographiques
\end{tabular}}

- Arnett J. J. 1997, Young people's conceptions of the transition to adulthood, Youth \& Society, vol. $29, \mathrm{n}^{\circ}$ 1:3-23.

- Bendit R., Hein K. et Biggart A., 2004, Delayed and negotiated autonomy. Domestic emancipation of young Europeans, DISKURS, $\mathrm{n}^{\circ}$ 3:76-85.

- Billari F. C. et Wilson C., 2001, "Convergence towards diversity? Cohort dynamics in the transition to adulthood in contemporary Western Europe », Working paper, WP 2001-039, Max Plank Institute for Demographic Research, Rostock.

- Billari F. C., Fürnkranz J. et Prskawetz A., 2000, "Timing, sequencing and quantum of life course events: a machine learning approach », Working paper, WP 2000-010, Max Plank Institute for Demographic Research, Rostock.

- Bourdieu P., 1982, Les rites comme actes d'institutions, Actes de la recherche en sciences sociales, $\mathrm{n}^{\circ} 43: 58-63$.

- Bradley H. et Devadason R., 2008, Fracture transitions: young adults' pathways into contemporary labour markets, Sociology, vol. 42, $\mathrm{n}^{\circ}$ 1:119-136.

- Brückner H. et Mayer K. U., 2005, De-standardization of the life course: what it might mean? And if it means anything, whether if actually took place?, in The structure of the life course: Standardized? individualized? differentiated? (sous la dir. de Macmillan R.) Advances in life course research (vol. 9), London: Elsevier:27-53.

- Cachón Rodríguez L., 2008, De las políticas de transitión en Europa a las clases de transiciones y transiciones de clase en España, Pensamiento Iberoamericano, n³:97-116.

- Cardoso A., 1997, Casar? Por que não? - Práticas e perspectivas de jovens portugueses sobre o casamento e a família, in Jovens em Mudança (sous la dir. de Chisholm L. et Pais J. M.), Lisboa, Imprensa de Ciências Sociais:201-221.

- Chambaz C., 2001, Les jeunes adultes en Europe: indépendance résidentielle, activité, ressources, Recherches et Prévisions, ${ }^{\circ}$ 65:53-71. 
- Chisholm L. et Pais J. M. (dir.), 1997, Jovens em Mudança, Lisboa, Imprensa de Ciências Sociais.

- Chisholm L., Büchner P., Krüger H. et Brown P. H. (dir.), 1998, Childhood, youth and social change: A comparative perspective, Londres: Falmer.

- Chisholm L., Büchner P., Krüger H. H. et du Bois-Reymond M. (dir.). 1995, Growing up in Europe. Contemporary Horizons in Childhood and Youth Studies. Berlin: de Gruyter.

- Cicchelli V., Pugeault-Cicchelli C. et Merico M., 2006, Individual and social temporalities in American sociology, Time \& Society, vol. 15, $\mathrm{n}^{\circ}$ 1:141-158.

- Cicchelli V. et Bréviglieri M. (dir.). 2007, Adolescences méditerranéennes. L'espace public à petits pas, Paris, Institut national de la Jeunesse et de l'Éducation populaire/L'Harmattan, Collection Débats Jeunesse.

- Cicchelli V., 2001, La dépendance familiale des jeunes adultes en France et en Italie, Recherches et Prévisions, $n^{\circ}$ 65:31-37.

- Deschavanne É. et Tavoillot P.-H., 2007, Philosophie des âges de la vie. Paris, Grasset.

- Du Bois-Reymond M. et López Blasco A., 2003, Yo-yo transitions and misleading trajectories: towards integrated transition policies for young adults in Europe, in Young people and contradictions of inclusion: towards Integrated Transition Policies in Europe (sous la dir. de López Blasco A., McNeish W. et Walther A.), Bristol, Policy Press.

- EGRIS, European Group for Integrated Social Research, 2001, Misleading trajectories: transitions dilemmas of young adults in Europe, Journal of Youth Studies, vol. 4, $\mathrm{n}^{\circ}$ 1:101-118.

- Esping-Andersen G., 1999, The social foundations of postindustrial economies, Oxford University Press.

- Esping-Andersen G., 1990, The three worlds of welfare capitalism, Cambridge: Polity Press \& Princeton, Princeton University Press.

- Ferreira V. S., Figueiredo A. et Lorga da Silva C., 1999, Jovens em Portugal. Análise longitudinal de fontes estatísticas (1960-1997), Oeiras, Celta/SEJ.

- Ferreira V. S. (dir.), 2006, A condição juvenil Portuguesa na viragem do milénio. Um retrato longitudinal através de fontes estatísticas oficiais: 1990-2005, Lisboa, Instituto Português da Juventude.

- Ferreira V. S., 2008, Mascas que demarcam: Tatuagem, body piercing e culturas juvenis, Lisboa, Imprensa de Ciências Sociais.

- Fonseca L. P. (da), 2001, Culturas juvenis, percursos femininos: experiências e subjectividades na educação de raparigas, Oeiras, Celta Editora.

- Fournier M. (dir.), 2008, Les âges de la vie bouleversés, Sciences humaines, n 193:26-55.

- Gauthier M., 2000, L'âge des jeunes : un "fait social » instable, Lien social et politiques, $n^{\circ} 43: 23-42$.

- Guillemard A.-M., 2005, The advent of a flexible life-course and the reconfiguration of welfare, in The changing face of welfare (sous la dir. de Gul Andersen J., Guillemard A.-M., Jensen P. et Pfau-Effinger B.), Manchester, Policy Press:53-72.

- Kovacheva S., 2001, Flexibilisation of youth transitions in central and eastern Europe, Young, vol. 9, $\mathrm{n}^{\circ} 1: 41-60$.

- Kugelberg C., 1998, Imagens culturais dos jovens suecos acerca do início da vida adulta; Sociologia-Problemas e Práticas, $n^{\circ}$ 27:41-57.

- Matsuo H., Symons K., Buellens K. et Billiet K., 2009, Response based quality assessment in the ESS - Round 3, Leuven, Center of Sociological Research (CeSO).

- Moen P., 1996, Gender, age, and the life course, in Handbook of aging and the social sciences (sous la dir. de Binstock R. H. et George L. K.), Thousand Oaks, CA, Sage Publications, Inc:171-187. 
- Moreno M., 2008, Rasgos característicos de la transición a la vida adulta de los jóvenes españoles en el marco comparado europeo, Pensamiento Iberoamericano, n 3:17-46.

- Müller W. et Gangl M. (dir.). 2003. Transitions from education to work in Europe: The integration of youth into EU labour markets, Oxford, New York, Oxford University Press.

- Neugarten B. L., Moore J. W. et Lowe J. C., 1965, Age norms, age constraints and adult socialization, American Journal of Sociology, $\mathrm{n}^{\circ}$ 70:710-17.

- Nugin R., 2008, Constructing adulthood in a world of uncertainties. Some case sof postwestern estonia, Young. Nordic Journal of Youth Research, $n^{\circ}$ 16:185-207.

- Pais J. M., 2009, A juventude como fase de vida: dos ritos de passagem aos ritos de impasse, Saúde e Sociedade, vol. 18, $n^{\circ} 3: 371-381$.

- Pais J. M., 2001, Ganchos, tachos e biscates. Jovens, trabalho e futuro, Porto, Âmbar.

- Pais J. M., 1996, A geração yô-yô, in Dinâmicas Multiculturais, Novas Faces, Outros Olhares (vol. II), Lisboa, ICS:111-125.

- Pais J. M., 1993, Culturas Juvenis, Lisboa, Imprensa Nacional/Casa da Moeda.

- Plug W., Zeil E. et Du Bois-Reymond M., 2003, Young peoples's perceptions on youth and adulthood. A longitudinal study from the Netherlands, Journal of Youth Studies, vol. 6, $\mathrm{n}^{\circ} 2: 127-144$.

- Shanahan M.- J., 2000, Pathways to adulthood in changing societies: variability and mechanisms in life course perspective, Annual Review of Sociology, ${ }^{\circ}$ 26:667-692.

- Silva Gomes (da) C., 1999, Escolhas escolares, heranças sociais: origens, expectativas e aspirações dos jovens no ensino secundário, Oeiras, Celta.

- Stauber B., 2006, Biography and gender in youth transitions, in The modernization of youth transitions in Europe (sous la dir. de Du Bois-Reymond M. et Chisholm L.), San Francisco, Jossey-Bass.

- Tomanovic S. et Ignjatovic S., 2006, Attitudes on transition to adulthood among young people in Serbia, Sociologija, vol. XLVIII, $\mathrm{n}^{\circ}$ 1:55-72.

- Van de Velde C., 2005, La entrada en la vida adulta. Una comparación europea, Revista de Estudios de Juventud, $\mathrm{n}^{\circ}$ 71:55-65.

- Vultur M., 2005, Las estructuras difuminadas de la edad adulta: transformación de las relaciones sociales y "prolongamiento de la juventud » en Rumanía, Revista de Estudios de Juventud, $\mathrm{n}^{\circ} 7181-94$.

- Walther A., 2006, Regimes of youth transitions. Choice, flexibility and security in young people's experiences across different European contexts, Young, vol. 14, n 1:119-141.

- Walther A. et Pohl A., 2005, «Thematic study on policy measures concerning disadvantaged youth», rapport final, Institute for Regional Innovation and Social Research (IRIS). http://ec.europa.eu/employment_social/social_inclusion/docs/youth_study_en.pdf.

- Walther A., Stauber B., Biggart A., du Bois-Reymond M., Furlong A., López Blasco A., Morch S. et Pais J. M. (dir.), 2002 a, Misleading trajectories - Integration policies for young adults in Europe?, Opladen, Leske:Budrich.

- Walther A., Hejl G. M. et Jensen T. B., 2002 b, « Youth transitions, youth policy and participation - State of the art report », Institute for Regional Innovation and Social Research (IRIS). http://www.iris-egris.de/yoyo/pdf/YoyoWP1StateofArt.pdf.

- Walther A., Stauber B., Bolay E., du Bois Reymond M., Mørch S., Pais J. M. et Schröer A., 1999, Young adults in Europe-new trajectories between youth and adulthood, in Intercultural reconstruction [sous la dir. de Circle for Youth Research Cooperation in Europe (CYRCE)], European Yearbook for Youth Policy and Research, vol. 2. Berlin et New York, Walther de Gruyter:61-89. 


\section{A N N EX E}

Tableau 1 - Âges de passage concernant différents marqueurs sociaux de transition vers l'âge adulte, et intervalles correspondant en années, par pays (moyennes)

\begin{tabular}{|c|c|c|c|c|c|c|c|c|c|}
\hline & $\begin{array}{l}\text { Premier } \\
\text { emploi }\end{array}$ & $\begin{array}{l}\text { Départ } \\
\text { du foyer } \\
\text { parental }\end{array}$ & $\begin{array}{l}\text { Intervalle } \\
\text { (années) }\end{array}$ & $\begin{array}{c}\text { Première } \\
\text { expérience de } \\
\text { vie en couple }\end{array}$ & $\begin{array}{l}\text { Intervalle } \\
\text { (années) }\end{array}$ & $\begin{array}{l}\text { Premier } \\
\text { mariage }\end{array}$ & $\begin{array}{l}\text { Intervalle } \\
\text { (années) }\end{array}$ & $\begin{array}{c}\text { Premier } \\
\text { enfant }\end{array}$ & $\begin{array}{l}\text { Intervalle } \\
\text { (années) }\end{array}$ \\
\hline Portugal & 17,7 & 21,6 & 3,9 & 23,5 & 1,9 & 23,6 & 0,1 & 25,4 & 1,8 \\
\hline Suisse & 17,9 & 20,2 & 2,3 & 24,4 & 4,2 & 26,8 & 2,4 & 27,7 & 0,9 \\
\hline Allemagne & 17,9 & 21,4 & 3,5 & 23,7 & 2,3 & 25,2 & 1,5 & 26,3 & 1,1 \\
\hline Autriche & 18,2 & 20,8 & 2,6 & 23,0 & 2,2 & 24,9 & 1,9 & 25,5 & 0,6 \\
\hline Danemark & 18,2 & 19,0 & 0,8 & 22,8 & 3,8 & 26,5 & 3,7 & 26,5 & 0,0 \\
\hline Finlande & 18,3 & 20,0 & 1,7 & 23,4 & 3,4 & 25,4 & 2,0 & 26,3 & 0,9 \\
\hline Royaume-Uni & 18,5 & 20,4 & 1,9 & 23,4 & 3,0 & 24,8 & 1,4 & 26,5 & 1,7 \\
\hline Hongrie & 18,7 & 21,9 & 3,2 & 22,4 & 0,5 & 22,8 & 0,4 & 24,2 & 1,4 \\
\hline Espagne & 18,8 & 21,7 & 2,9 & 25,3 & 3,6 & 25,7 & 0,4 & 27,1 & 1,4 \\
\hline Suède & 18,9 & 19,9 & 1,0 & 23,2 & 3,3 & 26,9 & 3,7 & 26,5 & $-0,4$ \\
\hline Ukraine & 19,0 & 20,1 & 1,1 & 22,6 & 2,5 & 22,7 & 0,1 & 23,9 & 1,2 \\
\hline Russie & 19,2 & 20,7 & 1,5 & 22,8 & 2,1 & 22,9 & 0,1 & 24,0 & 1,1 \\
\hline Pays-Bas & 19,3 & 21,7 & 2,4 & 24,0 & 2,3 & 25,6 & 1,6 & 27,4 & 1,8 \\
\hline Norvège & 19,4 & 19,4 & 0,0 & 23,5 & 4,1 & 25,6 & 2,1 & 26,0 & 0,4 \\
\hline Slovaquie & 19,7 & 21,8 & 2,1 & 22,8 & 1,0 & 22,9 & 0,1 & 23,9 & 1,0 \\
\hline France & 19,8 & 20,8 & 1,0 & 23,3 & 2,5 & 25,0 & 1,7 & 26,4 & 1,4 \\
\hline Pologne & 19,9 & 22,0 & 2,1 & 23,5 & 1,5 & 23,6 & 0,1 & 24,4 & 0,8 \\
\hline Belgique & 20,2 & 22,5 & 2,3 & 23,5 & 1,0 & 24,2 & 0,7 & 26,2 & 2,0 \\
\hline Ireland & 20,2 & 21,3 & 1,1 & 25,4 & 4,1 & 26,4 & 1,0 & 27,2 & 0,8 \\
\hline Chypre & 20,3 & 21,1 & 0,8 & 23,8 & 2,7 & 24,2 & 0,4 & 25,4 & 1,2 \\
\hline Bulgarie & 20,4 & 20,8 & 0,4 & 21,9 & 1,1 & 22,1 & 0,2 & 23,6 & 1,5 \\
\hline Slovénie & 20,6 & 23,4 & 2,8 & 23,7 & 0,3 & 24,4 & 0,7 & 25,1 & 0,7 \\
\hline Estonie & 21,4 & 19,8 & $-1,6$ & 23,2 & 3,4 & 23,9 & 0,7 & 24,7 & 0,8 \\
\hline Total & 19,0 & 21,1 & 2,1 & 23,4 & 2,3 & 24,2 & 0,8 & 25,4 & 1,2 \\
\hline
\end{tabular}

Source: European Social Survey, 2006.

Tableau 2 - Répondants entre 15 et 29 ans, par pays, qui ont déjà... (en \%)

\begin{tabular}{|c|c|c|c|c|c|}
\hline & Eu un emploi & $\begin{array}{c}\text { Quitté le foyer } \\
\text { parental }\end{array}$ & Vécu en couple & Été mariés & Eu des enfants \\
\hline Slovénie & 42,2 & 27,9 & 9,3 & 3,5 & 9,6 \\
\hline Bulgarie & 47,6 & 29,0 & 12,2 & 18,3 & 26,2 \\
\hline Portugal & 48,8 & 26,9 & 6,0 & 11,9 & 12,9 \\
\hline Slovaquie & 49,0 & 35,9 & 8,6 & 14,3 & 13,4 \\
\hline France & 55,1 & 51,0 & 19,7 & 11,9 & 13,0 \\
\hline Ukraine & 56,4 & 33,1 & 13,5 & 33,9 & 26,5 \\
\hline Autriche & 56,7 & 31,7 & - & 3,3 & 5,1 \\
\hline Belgique & 57,3 & 32,1 & 10,5 & 7,1 & 10,4 \\
\hline Pologne & 57,8 & 28,6 & 10,4 & 19,0 & 15,8 \\
\hline Estonie & 58,1 & 49,3 & 18,5 & 8,2 & 13,8 \\
\hline Russie & 60,9 & 37,6 & 15,0 & 20,3 & 21,6 \\
\hline Norvège & 61,8 & 64,7 & 20,1 & 7,9 & 14,0 \\
\hline Suède & 62,3 & 63,1 & 25,0 & 7,4 & 11,2 \\
\hline Hongrie & 62,6 & 36,0 & 8,2 & 15,9 & 17,6 \\
\hline Irlande & 65,2 & 55,0 & 17,0 & 7,1 & 13,9 \\
\hline Allemagne & 65,3 & 47,3 & 15,4 & 9,5 & 9,7 \\
\hline Chypre & 66,5 & 35,7 & 11,8 & 11,9 & 8,1 \\
\hline Royaume-Uni & 66,7 & 53,7 & 16,5 & 9,7 & 15,4 \\
\hline Pays-Bas & 67,5 & 51,6 & 12,5 & 12,0 & 8,4 \\
\hline Finlande & 67,7 & 66,4 & - & 12,2 & 14,0 \\
\hline Danemark & 69,0 & 68,2 & 21,4 & 8,5 & 10,0 \\
\hline Espagne & 70,6 & 33,6 & 9,4 & 8,1 & 9,5 \\
\hline Suisse & 73,1 & 45,2 & 14,4 & 10,8 & 6,9 \\
\hline Total & 60,1 & 42,8 & 13,8 & 11,8 & 13,8 \\
\hline
\end{tabular}

Source: European Social Survey, 2006.

Aucune donnée n'est disponible concernant la vie en couple pour l'Autriche et la Finlande. 